\title{
Approximating common fixed points in hyperbolic spaces
}

\author{
Hafiz Fukhar-ud-din ${ }^{1,2}$ and Mohamed Amine Khamsi ${ }^{3 *}$
}

\section{"Correspondence:}

mohamed@math.utep.edu

${ }^{3}$ Department of Mathematical Sciences, University of Texas at El Paso, El Paso, TX 79968, USA Full list of author information is available at the end of the article

\begin{abstract}
We establish strong convergence and $\Delta$-convergence theorems of an iteration scheme associated to a pair of nonexpansive mappings on a nonlinear domain. In particular we prove that such a scheme converges to a common fixed point of both mappings. Our results are a generalization of well-known similar results in the linear setting. In particular, we avoid assumptions such as smoothness of the norm, necessary in the linear case.

MSC: Primary 47H09; secondary 46B20; 47H10; 47E10

Keywords: $\Delta$-convergence; fixed point; Ishikawa iterations; nonexpansive mapping; strong convergence; uniformly convex hyperbolic space
\end{abstract}

\section{Introduction}

Let $C$ be a nonempty subset of a metric space $(X, d)$ and $T: C \rightarrow C$ be a mapping. Denote the set of fixed points of $T$ by $F(T)$. Then $T$ is (i) nonexpansive if $d(T x, T y) \leq d(x, y)$ for $x, y \in C$ (ii) quasi-nonexpansive if $F(T) \neq \emptyset$ and $d(T x, y) \leq d(x, y)$ for $x \in C$ and $y \in F(T)$. For an initial value $x_{1} \in C$, Das and Debata [1] studied the strong convergence of Ishikawa iterates $\left\{x_{n}\right\}$ defined by

$$
x_{n+1}=\alpha_{n} S\left(\beta_{n} T x_{n}+\left(1-\beta_{n}\right) x_{n}\right)+\left(1-\alpha_{n}\right) x_{n}
$$

for two quasi-nonexpansive mappings $S, T$ on a nonempty closed and convex subset of a strictly convex Banach space. Takahashi and Tamura [2] proved weak convergence of (1.1) to a common fixed point of two nonexpansive mappings in a uniformly convex Banach space which satisfies Opial's condition or whose norm is Fréchet differentiable and strong convergence in a strictly convex Banach space (see also [3, 4]). Mann and Ishikawa iterative procedures are well-defined in a vector space through its built-in convexity. In the literature, several mathematicians have introduced the notion of convexity in metric spaces; for example [5-8]. In this work, we follow the original metric convexity introduced by Menger [9] and used by many authors like Kirk [5, 6] and Takahashi [8]. Note that Mann iterative procedures were also investigated in hyperbolic metric spaces $[10,11]$.

In this paper we investigate the results published in [2] and generalize them to uniformly convex hyperbolic spaces. A particular example of such metric spaces is the class of CAT(0)-spaces (in the sense of Gromov) and $\mathbb{R}$-trees (in the sense of Tits). Heavy use of the linear structure of Banach spaces in [2] presents some difficulties when extending 
these results to metric spaces. For example, a key assumption in many of their results is the smoothness of the norm which is hard to extend to metric spaces.

\section{Menger convexity in metric spaces}

Let $(X, d)$ be a metric space. Assume that for any $x$ and $y$ in $X$, there exists a unique metric segment $[x, y]$, which is an isometric copy of the real line interval $[0, d(x, y)]$. Note by $\mathcal{F}$ the family of the metric segments in $X$. For any $\beta \in[0,1]$, there exists a unique point $z \in[x, y]$ such that

$$
d(x, z)=(1-\beta) d(x, y) \quad \text { and } \quad d(z, y)=\beta d(x, y) .
$$

Throughout this paper we will denote such point by $\beta x \oplus(1-\beta) y$. Such metric spaces are usually called convex metric spaces [9]. Moreover, if we have

$$
d(\alpha p \oplus(1-\alpha) x, \alpha q \oplus(1-\alpha) y) \leq \alpha d(p, q)+(1-\alpha) d(x, y)
$$

for all $p, q, x, y$ in $X$ and $\alpha \in[0,1]$, then $X$ is said to be a hyperbolic metric space (see [1113]). For $q=y$, the hyperbolic inequality reduces to the convex structure inequality [8]. Throughout this paper, we will assume

$$
\alpha x \oplus(1-\alpha) y=(1-\alpha) y \oplus \alpha x
$$

for any $\alpha \in[0,1]$ and any $x, y \in X$.

An example of hyperbolic spaces is the family of Banach vector spaces or any normed vector spaces. Hadamard manifolds [14], the Hilbert open unit ball equipped with the hyperbolic metric [15], and the CAT(0) spaces [6,16-20] (see Example 2.1) are examples of nonlinear structures which play a major role in recent research in metric fixed point theory. A subset $C$ of a hyperbolic space $X$ is said to be convex if $[x, y] \subset C$, whenever $x, y \in C$ (see also [21]).

Definition 2.1 [22,23] Let $(X, d)$ be a hyperbolic metric space. For any $r>0$ and $\varepsilon>0$, set

$$
\delta(r, \varepsilon)=\inf \left\{1-\frac{1}{r} d\left(\frac{1}{2} x \oplus \frac{1}{2} y, a\right) ; d(x, a) \leq r, d(y, a) \leq r, d(x, y) \geq r \varepsilon\right\}
$$

for any $a \in X$. X is said to be uniformly convex whenever $\delta(r, \varepsilon)>0$, for any $r>0$ and $\varepsilon>0$.

Throughout this paper we assume that if $X$ is a uniformly convex hyperbolic space, then for every $s \geq 0$ and $\varepsilon>0$, there exists $\eta(s, \varepsilon)>0$ such that

$$
\delta(r, \varepsilon)>\eta(s, \varepsilon)>0 \quad \text { for any } r>s .
$$

\section{Remark 2.1}

(i) We have $\delta(r, 0)=0$. Moreover, $\delta(r, \varepsilon)$ is an increasing function of $\varepsilon$.

(ii) For $r_{1} \leq r_{2}$, we have

$$
1-\frac{r_{2}}{r_{1}}\left(1-\delta\left(r_{2}, \varepsilon \frac{r_{1}}{r_{2}}\right)\right) \leq \delta\left(r_{1}, \varepsilon\right) .
$$


Next we give a very important example of uniformly convex hyperbolic metric space.

Example 2.1 [16] Let $(X, d)$ be a metric space. A geodesic from $x$ to $y$ in $X$ is a mapping $c$ from a closed interval $[0, l] \subset \mathbb{R}$ to $X$ such that $c(0)=x, c(l)=y$, and $d\left(c(t), c\left(t^{\prime}\right)\right)=\left|t-t^{\prime}\right|$ for all $t, t^{\prime} \in[0, l]$. In particular, $c$ is an isometry and $d(x, y)=l$. The image $\alpha$ of $c$ is called a geodesic (or metric) segment joining $x$ and $y$. The space $(X, d)$ is said to be a geodesic space if every two points of $X$ are joined by a geodesic and $X$ is said to be uniquely geodesic if there is exactly one geodesic joining $x$ and $y$ for each $x, y \in X$, which will be denoted by $[x, y]$, and called the segment joining $x$ to $y$.

A geodesic triangle $\Delta\left(x_{1}, x_{2}, x_{3}\right)$ in a geodesic metric space $X$ consists of three points $x_{1}$, $x_{2}, x_{3}$ in $X$ (the vertices of $\Delta$ ) and a geodesic segment between each pair of vertices (the edges of $\Delta)$. A comparison triangle for geodesic triangle $\Delta\left(x_{1}, x_{2}, x_{3}\right)$ in $(X, d)$ is a triangle $\bar{\Delta}\left(x_{1}, x_{2}, x_{3}\right):=\Delta\left(\bar{x}_{1}, \bar{x}_{2}, \bar{x}_{3}\right)$ in $\mathbb{R}^{2}$ such that $d_{\mathbb{R}^{2}}\left(\bar{x}_{i}, \bar{x}_{j}\right)=d\left(x_{i}, x_{j}\right)$ for $i, j \in\{1,2,3\}$. Such a triangle always exists (see [18]).

A geodesic metric space is said to be a $\mathrm{CAT}(0)$ space if all geodesic triangles of appropriate size satisfy the following $\mathrm{CAT}(0)$ comparison axiom.

Let $\Delta$ be a geodesic triangle in $X$ and let $\bar{\Delta} \subset \mathbb{R}^{2}$ be a comparison triangle for $\Delta$. Then $\Delta$ is said to satisfy the $\mathrm{CAT}(0)$ inequality if for all $x, y \in \Delta$ and all comparison points $\bar{x}, \bar{y} \in \bar{\Delta}$,

$$
d(x, y) \leq d(\bar{x}, \bar{y}) .
$$

Complete CAT(0) spaces are often called Hadamard spaces (see [16]). If $x, y_{1}, y_{2}$ are points of a $\mathrm{CAT}(0)$ space, then the $\mathrm{CAT}(0)$ inequality implies

$$
d^{2}\left(x, \frac{1}{2} y_{1} \oplus \frac{1}{2} y_{2}\right) \leq \frac{1}{2} d^{2}\left(x, y_{1}\right)+\frac{1}{2} d^{2}\left(x, y_{2}\right)-\frac{1}{4} d^{2}\left(y_{1}, y_{2}\right) .
$$

The above inequality is known as the $(\mathrm{CN})$ inequality of Bruhat and Tits [24]. The $(\mathrm{CN})$ inequality implies that $\mathrm{CAT}(0)$ spaces are uniformly convex with

$$
\delta(r, \varepsilon)=1-\sqrt{1-\frac{\varepsilon^{2}}{4}} .
$$

In a hyperbolic space $X,(1.1)$ is written as

$$
x_{n+1}=\alpha_{n} S\left(\beta_{n} T x_{n} \oplus\left(1-\beta_{n}\right) x_{n}\right) \oplus\left(1-\alpha_{n}\right) x_{n},
$$

where $\alpha_{n}, \beta_{n} \in[0,1]$. If $S=T$ in (2.1), it reduces to the following Ishikawa iteration process of one mapping:

$$
x_{n+1}=\alpha_{n} T\left(\beta_{n} T x_{n} \oplus\left(1-\beta_{n}\right) x_{n}, n \geq 1,\right) \oplus\left(1-\alpha_{n}\right) x_{n}
$$

where $\alpha_{n}, \beta_{n} \in[0,1]$. Let $\left\{x_{n}\right\}$ be a bounded sequence in a metric space $X$ and $C$ be a nonempty subset. Define $r\left(\cdot,\left\{x_{n}\right\}\right): C \rightarrow[0, \infty)$, by

$$
r\left(x,\left\{x_{n}\right\}\right)=\limsup _{n \rightarrow \infty} d\left(x, x_{n}\right)
$$


The asymptotic radius $\rho_{C}$ of $\left\{x_{n}\right\}$ with respect to $C$ is given by

$$
\rho_{C}=\inf \left\{r\left(x,\left\{x_{n}\right\}\right): x \in C\right\}
$$

$\rho$ will denote the asymptotic radius of $\left\{x_{n}\right\}$ with respect to $X$. A point $\xi \in C$ is said to be an asymptotic center of $\left\{x_{n}\right\}$ with respect to $C$ if $r\left(\xi,\left\{x_{n}\right\}\right)=r\left(C,\left\{x_{n}\right\}\right)=\min \left\{r\left(x,\left\{x_{n}\right\}\right): x \in C\right\}$. We denote with $A\left(C,\left\{x_{n}\right\}\right)$, the set of asymptotic centers of $\left\{x_{n}\right\}$ with respect to $C$. When $C=X$, we call $\xi$ an asymptotic center of $\left\{x_{n}\right\}$ and we use the notation $A\left(\left\{x_{n}\right\}\right)$ instead of $A\left(X,\left\{x_{n}\right\}\right)$. In general, the set $A\left(C,\left\{x_{n}\right\}\right)$ of asymptotic centers of a bounded sequence $\left\{x_{n}\right\}$ may be empty or may even contain infinitely many points. Note that in the study of the geometry of Banach spaces, the function $r\left(\cdot,\left\{x_{n}\right\}\right)$ is also known as a type. For more on types in metric spaces, we refer to [25].

The $\Delta$-convergence, introduced independently several years ago by Kuczumow [26] and Lim [27], is shown in CAT(0) spaces to behave similarly as the weak convergence in Banach spaces.

Definition 2.2 A bounded sequence $\left\{x_{n}\right\}$ in $X$ is said to $\Delta$-converge to $x \in X$ if $x$ is the unique asymptotic center of every subsequence $\left\{u_{n}\right\}$ of $\left\{x_{n}\right\}$. We write $x_{n} \stackrel{\Delta}{\rightarrow} x\left(\left\{x_{n}\right\}\right.$ $\Delta$-converges to $x$ ).

In this paper, we study the iteration schemes (2.1)-(2.2) for nonexpansive mappings. We study strong convergence of these iterates in strictly convex hyperbolic spaces and prove $\Delta$-convergence results in uniformly convex hyperbolic spaces without requiring any condition similar to norm Fréchet differentiability.

In the sequel, the following results will be needed.

Lemma 2.1 $[25,28]$ Let $X$ be a hyperbolic metric spaces. Assume that $X$ is uniformly convex. Let $C$ be a nonempty, closed and convex subset of $X$. Then every bounded sequence $\left\{x_{n}\right\} \in X$ has a unique asymptotic center with respect to $C$.

Lemma $2.2[25,28]$ Let $X$ be a hyperbolic metric spaces. Assume that $X$ is uniformly convex. Let $C$ be a nonempty, closed and convex subset of $X$. Let $C$ be a nonempty closed and convex subset of $X$, and $\left\{x_{n}\right\}$ be a bounded sequence in $C$ such that $A\left(\left\{x_{n}\right\}\right)=\{y\}$ and $r\left(\left\{x_{n}\right\}\right)=\rho$. If $\left\{y_{m}\right\}$ is a sequence in C such that $\lim _{m \rightarrow \infty} r\left(y_{m},\left\{x_{n}\right\}\right)=\rho$, then $\lim _{m \rightarrow \infty} y_{m}=y$.

The following lemma [29] will be useful in studying the sequence generated by (2.1) in uniformly convex metric spaces. Here we give a proof based on the ideas developed in [25].

Lemma 2.3 Let $X$ be a uniformly convex hyperbolic space. Then for arbitrary positive numbers $\varepsilon>0$ and $r>0$, and $\alpha \in[0,1]$, we have

$$
d(a, \alpha x \oplus(1-\alpha) y) \leq r(1-\delta(r, 2 \min \{\alpha, 1-\alpha\} \varepsilon))
$$

for all $a, x, y \in X$, such that $d(z, x) \leq r, d(z, y) \leq r$, and $d(x, y) \geq r \varepsilon$

Proof Without loss of generality, we may assume $\alpha<\frac{1}{2}$. In this case, we have $\min \{\alpha, 1-$ $\alpha\}=\alpha$. Let $a \in X$ be fixed and $x, y \in X$. Set $\bar{x}=2 \alpha x \oplus(1-2 \alpha) y$. Since

$$
d\left(\frac{1}{2} \bar{x} \oplus \frac{1}{2} y, x\right) \leq(1-\alpha) d(x, y) \quad \text { and } \quad d\left(\frac{1}{2} \bar{x} \oplus \frac{1}{2} y, y\right)=\alpha d(x, y),
$$


the uniform convexity of $X$ will imply $\frac{1}{2} \bar{x} \oplus \frac{1}{2} y=\alpha x \oplus(1-\alpha) y$. Using the uniform convexity of $X$, we get

$$
d\left(a, \frac{1}{2} \bar{x} \oplus \frac{1}{2} y\right) \leq r(1-\delta(r, 2 \alpha \varepsilon)) .
$$

Hence

$$
d(a, \alpha x \oplus(1-\alpha) y) \leq r(1-\delta(r, 2 \min \{\alpha, 1-\alpha\} \varepsilon)) .
$$

Remark 2.2 If $(X, d)$ is uniformly convex, then $(X, d)$ is strictly convex, i.e., whenever

$$
d(\alpha x \oplus(1-\alpha) y, a)=d(x, a)=d(y, a)
$$

for $\alpha \in(0,1)$ and any $x, y, a \in X$, then we must have $x=y$.

The following result is very useful.

Lemma 2.4 [25] Let $(X, d)$ be a uniformly convex hyperbolic space. Let $R \in[0,+\infty)$ be such that

$$
\limsup _{n \rightarrow \infty} d\left(x_{n}, a\right) \leq R, \quad \limsup _{n \rightarrow \infty} d\left(y_{n}, a\right) \leq R \quad \text { and } \quad \lim _{n \rightarrow \infty} d\left(a, \frac{1}{2} x_{n} \oplus \frac{1}{2} y_{n}\right)=R .
$$

Then we have

$$
\lim _{n \rightarrow \infty} d\left(x_{n}, y_{n}\right)=0
$$

But since we use convex combinations other than the middle point, we will need the following generalization obtained by using Lemma 2.3 .

Lemma 2.5 Let $(X, d)$ be a uniformly convex hyperbolic space. Let $R \in[0,+\infty)$ be such that $\limsup _{n \rightarrow \infty} d\left(x_{n}, a\right) \leq R, \lim \sup _{n \rightarrow \infty} d\left(y_{n}, a\right) \leq R$, and

$$
\lim _{n \rightarrow \infty} d\left(a, \alpha_{n} x_{n} \oplus\left(1-\alpha_{n}\right) y_{n}\right)=R,
$$

where $\alpha_{n} \in[a, b]$, with $0<a \leq b<1$. Then we have

$$
\lim _{n \rightarrow \infty} d\left(x_{n}, y_{n}\right)=0
$$

A subset $C$ of a metric space $X$ is Chebyshev if for every $x \in X$, there exists $c_{0} \in C$ such that $d\left(c_{0}, x\right)<d(c, x)$ for all $c \in C, c \neq c_{0}$. In other words, for each point of the space, there is a well-defined nearest point of $C$. We can then define the nearest point projection $P$ : $X \rightarrow C$ by sending $x$ to $c_{0}$. We have the following result.

Lemma 2.6 [25] Let $(X, d)$ be a complete uniformly convex hyperbolic space. Let $C$ be nonempty, convex and closed subset of $X$. Let $x \in X$ be such that $d(x, C)<\infty$. Then there exists a unique best approximant of $x$ in $C$, i.e., there exists a unique $c_{0} \in C$ such that

$$
d\left(x, c_{0}\right)=d(x, C)=\inf \{d(x, c) ; c \in C\},
$$




\section{Convergence in strictly convex hyperbolic space}

Let $(X, d)$ be a hyperbolic metric space. Let $C$ be a nonempty closed convex subset of $X$. Let $S, T: C \rightarrow C$ be two nonexpansive mappings. Throughout the paper, assume that $F=$ $F(S) \cap F(T)$. Let $x_{1} \in C$ and $p \in F$ (assuming $F$ is not empty). Set $r=d\left(x_{1}, p\right)$. Then

$$
C\left(x_{1}\right)=C \cap B(p, r)=\{x \in C ; d(p, x) \leq r\}
$$

is nonempty and invariant by both $S$ and $T$. Therefore one may always assume that $C$ is bounded provided that $S$ and $T$ have a common fixed point. Moreover, if $\left\{x_{n}\right\}$ is the sequence generated by (2.1), then we have

$$
\begin{aligned}
d\left(x_{n+1}, p\right) & =d\left(\alpha_{n} S y_{n} \oplus\left(1-\alpha_{n}\right) x_{n}, p\right) \\
& \leq \alpha_{n} d\left(S y_{n}, p\right)+\left(1-\alpha_{n}\right) d\left(x_{n}, p\right) \\
& \leq \alpha_{n} d\left(y_{n}, p\right)+\left(1-\alpha_{n}\right) d\left(x_{n}, p\right) \\
& =\alpha_{n} d\left(\beta_{n} T x_{n} \oplus\left(1-\beta_{n}\right) x_{n}, p\right)+\left(1-\alpha_{n}\right) d\left(x_{n}, p\right) \\
& \leq \alpha_{n}\left[\beta_{n} d\left(T x_{n}, p\right)+\left(1-\beta_{n}\right) d\left(x_{n}, p\right)\right]+\left(1-\alpha_{n}\right) d\left(x_{n}, p\right) \\
& \leq d\left(x_{n}, p\right),
\end{aligned}
$$

where $y_{n}=\beta_{n} T x_{n} \oplus\left(1-\beta_{n}\right) x_{n}$. This proves that $\left\{d\left(x_{n}, p\right)\right\}$ is decreasing, which implies that $\lim _{n \rightarrow \infty} d\left(x_{n}, p\right)$ exists. Using the above inequalities, we get

$$
\begin{aligned}
\lim _{n \rightarrow \infty} d\left(x_{n}, p\right) & =\lim _{n \rightarrow \infty} d\left(\alpha_{n} S y_{n} \oplus\left(1-\alpha_{n}\right) x_{n}, p\right) \\
& =\lim _{n \rightarrow \infty} \alpha_{n} d\left(S y_{n}, p\right)+\left(1-\alpha_{n}\right) d\left(x_{n}, p\right) \\
& =\lim _{n \rightarrow \infty} \alpha_{n} d\left(y_{n}, p\right)+\left(1-\alpha_{n}\right) d\left(x_{n}, p\right) \\
& =\lim _{n \rightarrow \infty} \alpha_{n} d\left(\beta_{n} T x_{n} \oplus\left(1-\beta_{n}\right) x_{n}, p\right)+\left(1-\alpha_{n}\right) d\left(x_{n}, p\right) \\
& =\lim _{n \rightarrow \infty} \alpha_{n}\left[\beta_{n} d\left(T x_{n}, p\right)+\left(1-\beta_{n}\right) d\left(x_{n}, p\right)\right]+\left(1-\alpha_{n}\right) d\left(x_{n}, p\right) .
\end{aligned}
$$

The first result of this work discusses the convergence behavior of the sequence generated by (2.1).

Theorem 3.1 Let $X$ be a strictly convex hyperbolic space. Let $C$ be a nonempty bounded, closed and convex subset of $X$. Let $S, T: C \rightarrow C$ be two nonexpansive mappings. Assume that $F \neq \emptyset$. Let $x_{1} \in C$ and $\left\{x_{n}\right\}$ be given by (2.1). Then the following hold:

(i) if $\alpha_{n} \in[a, b]$ and $\beta_{n} \in[0, b]$, with $0<a \leq b<1$, then $x_{n_{i}} \rightarrow y$ implies $y \in F(S)$;

(ii) if $\alpha_{n} \in[a, 1]$ and $\beta_{n} \in[a, b]$, with $0<a \leq b<1$, then $x_{n_{i}} \rightarrow y$ implies $y \in F(T)$;

(iii) if $\alpha_{n}, \beta_{n} \in[a, b]$, with $0<a \leq b<1$, then $x_{n_{i}} \rightarrow y$ implies $y \in F$. In this case, we have $x_{n} \rightarrow y$.

Proof Assume that $x_{n_{i}} \rightarrow y$. Let $p \in F$. Without loss of generality, we may assume $\lim _{n \rightarrow \infty} \alpha_{n_{i}}=\alpha$ and $\lim _{n \rightarrow \infty} \beta_{n_{i}}=\beta$. Since $\left\{d\left(x_{n}, p\right)\right\}$ is decreasing, we get

$$
\lim _{n_{i} \rightarrow \infty} d\left(x_{n}, p\right)=\lim _{n_{i} \rightarrow \infty} d\left(x_{n_{i}}, p\right)=d(y, p)
$$


The above inequalities imply

$$
\begin{aligned}
d(y, p) & =d(\alpha S(\beta T y \oplus(1-\beta) y) \oplus(1-\alpha) y, p) \\
& =\alpha d(S(\beta T y \oplus(1-\beta) y), p)+(1-\alpha) d(y, p) \\
& =\alpha d(\beta T y \oplus(1-\beta) y, p)+(1-\alpha) d(y, p) \\
& =\alpha[\beta d(T y, p)+(1-\beta) d(y, p)]+(1-\alpha) d(y, p) .
\end{aligned}
$$

Set $r=d(y, p)$. Without loss of generality we may assume $r>0$ otherwise most of the conclusions in the theorem are trivial. Assume that $\liminf _{n \rightarrow \infty} \alpha_{n}>0$. Then $\alpha \neq 0$. Hence

$$
d(S(\beta T y \oplus(1-\beta) y), p)=d(\beta T y \oplus(1-\beta) y, p)=\beta d(T y, p)+(1-\beta) r=r
$$

which implies $\beta d(T y, p)=\beta r$. If we assume that $\liminf _{n \rightarrow \infty} \beta_{n}>0$, then $\beta \neq 0$, which implies $d(T y, p)=r$.

(1) If $\alpha \in(0,1)$ and $\beta>0$, then

$$
d(p, S(\beta T y \oplus(1-\beta) y))=d(\alpha S(\beta T y \oplus(1-\beta) y) \oplus(1-\alpha) y, p)=r .
$$

The strict convexity of $X$ will imply $S(\beta T y \oplus(1-\beta) y)=y$.

(2) If $\alpha \in(0,1)$ and $\beta=0$, then

$$
d(p, y)=d(p, S(y))=d(\alpha S(y) \oplus(1-\alpha) y, p) .
$$

The strict convexity of $X$ will imply $S(y)=y$.

(3) If $\beta \in(0,1)$ and $\alpha>0$, then

$$
d(p, y)=d(p, T(y))=d(p, \beta T y \oplus(1-\beta) y) .
$$

The strict convexity of $X$ will imply $T(y)=y$.

(4) If $\alpha, \beta \in(0,1)$, then $T(y)=y$ and $S(\beta T y \oplus(1-\beta) y)=y$. Hence $S(y)=y$.

Let us finish the proof of Theorem 3.1. Note that (i) implies $\alpha \in[a, b]$ and $\beta \in[0, b]$. If $\beta=0$, then the conclusion (2) above implies $y \in F(S)$. Otherwise the conclusion (4) will imply $y \in F$. This proves (i).

For (ii), notice that $\alpha \in[a, 1]$ and $\beta \in[a, b]$. Hence the conclusion (3) will imply $y \in F(T)$ which proves (ii).

For (iii), notice that $\alpha, \beta \in[a, b]$. Hence the conclusion (4) will imply $y \in F(T)$. Since

$$
\lim _{n \rightarrow \infty} d\left(y_{n}, y\right)=\lim _{n \rightarrow \infty} d\left(y_{n_{i}}, y\right)=0
$$

we get $x_{n} \rightarrow y$, which completes the proof of (iii).

If we assume compactness, Theorem 3.1 will imply the following result.

Theorem 3.2 Let $X$ be a strictly convex hyperbolic space. Let $C$ be a nonempty bounded, closed and convex subset of $X$. Let $S, T: C \rightarrow C$ be two nonexpansive mappings. Assume 
that $F \neq \emptyset$. Fix $x_{1} \in C$. Assume that $\overline{\mathrm{co}}\left\{\left\{x_{1}\right\} \cup S(C) \cup T(C)\right\}$ is a compact subset of C. Define $\left\{x_{n}\right\}$ as in (2.1) where $\alpha_{n}, \beta_{n} \in[a, b]$, with $0<a \leq b<1$, and $x_{1}$ is the initial element of the sequence. Then $\left\{x_{n}\right\}$ converges strongly to a common fixed point of $S$ and $T$.

Proof We have $x_{n} \in \overline{\mathrm{co}}\left\{\left\{x_{1}\right\} \cup S(C) \cup T(C)\right\}$. Since $\overline{\mathrm{co}}\left\{\left\{x_{1}\right\} \cup S(C) \cup T(C)\right\}$ is compact, $\left\{x_{n}\right\}$ has a convergent subsequence $\left\{x_{n_{i}}\right\}$, i.e., $x_{n_{i}} \rightarrow z$. By Theorem 3.1, we have $z \in F$ and $x_{n} \rightarrow z$.

The existence of a common fixed point $T$ and $S$ is crucial. If one assumes that $T$ and $S$ commute, i.e., $S \circ T=T \circ S$, then a common fixed point exists under the assumptions of Theorem 3.2. Indeed, fix $x_{0} \in C$ and define

$$
T_{n} x=\frac{1}{n} x_{0} \oplus\left(1-\frac{1}{n}\right) T x
$$

for $x \in C$ and $n \geq 1$. Then

$$
\begin{aligned}
d\left(T_{n} x, T_{n} y\right) & =d\left(\frac{1}{n} x_{0} \oplus\left(1-\frac{1}{n}\right) T x, \frac{1}{n} x_{0} \oplus\left(1-\frac{1}{n}\right) T y\right) \\
& \leq\left(1-\frac{1}{n}\right) d(T x, T y) \\
& \leq\left(1-\frac{1}{n}\right) d(x, y) .
\end{aligned}
$$

That is, $T_{n}$ is a contraction. By the Banach contraction principle (BCP), $T_{n}$ has a unique fixed point $u_{n}$ in $C$. Since the closure of $T(C)$ is compact, there exists a subsequence $\left\{T u_{n_{i}}\right\}$ of $\left\{T u_{n}\right\}$ such that $T u_{n_{i}} \rightarrow u$. Since $T(C)$ is bounded and

$$
d\left(u_{n}, T u_{n}\right)=d\left(\frac{1}{n} x_{0} \oplus\left(1-\frac{1}{n}\right) T u_{n}, T u_{n}\right) \leq \frac{1}{n} d\left(x_{0}, T u_{n}\right),
$$

we have $d\left(u_{n}, T u_{n}\right) \rightarrow 0$. In particular, we have $u_{n_{i}} \rightarrow u$. Continuity of $T$ implies $T u=u$. Since $X$ is strictly convex, then $F(T)$ is a nonempty convex subset of $X$. Since $T$ and $S$ commute, we have $S(F(T)) \subset F(T)$. Moreover, since the closure of $T(C)$ is compact, we see that $F(T)$ is compact. The above proof shows that $S$ has a fixed point in $F(T)$, i.e., $F=F(S) \cap F(T) \neq \emptyset$.

The case $S=T$ gives the following result.

Theorem 3.3 Let $C$ be a nonempty closed and convex subset of a complete strictly convex hyperbolic space $X$. Let $T: C \rightarrow C$ be a nonexpansive mapping such that $\overline{\mathrm{co}}\left\{\left\{c_{0}\right\} \cup T(C)\right\}$ is a compact subset of $C$, where $c_{0} \in C$. Define $\left\{x_{n}\right\}$ by (2.2), where $x_{1}=c_{0}, \alpha_{n} \in[a, b]$ and $\beta_{n} \in[0, b]$ or $\alpha_{n} \in[a, 1]$ and $\beta_{n} \in[a, b]$, with $0<a \leq b<1$. Then $\left\{x_{n}\right\}$ converges strongly to a fixed point of $T$.

Proof We saw that in this case, we have $F(T) \neq \emptyset$. Since $x_{n} \in \overline{c o}\left\{\left\{x_{1}\right\} \cup T(C)\right\}$. Then there exists a subsequence $\left\{x_{n_{i}}\right\}$ of $\left\{x_{n}\right\}$ such that $x_{n_{i}} \rightarrow z \in C$. By Theorem 3.1, we have $T z=z$ and $x_{n} \rightarrow z$. 


\section{Convergence in uniformly convex hyperbolic spaces}

The following result is similar to the well-known demi-closedness principle discovered by Göhde in uniformly convex Banach spaces [30].

Lemma 4.1 Let $C$ be a nonempty, closed and convex subset of a complete uniformly convex hyperbolic space X. Let $T: C \rightarrow C$ be a nonexpansive mapping. Let $\left\{x_{n}\right\} \in C$ be an approximate fixed point sequence of $T$, i.e., $\lim _{n \rightarrow \infty} d\left(x_{n}, T x_{n}\right)=0$. If $x \in C$ is the asymptotic center of $\left\{x_{n}\right\}$ with respect to $C$, then $x$ is a fixed point of $T$, i.e., $x \in F(T)$. In particular, if $\left\{x_{n}\right\} \in C$ is an approximate fixed point sequence of $T$, such that $x_{n} \stackrel{\Delta}{\rightarrow} x$, then $x \in F(T)$.

Proof Let $\left\{x_{n}\right\}$ be an approximate fixed point sequence of $T$. Let $x \in C$ be the unique asymptotic center of $\left\{x_{n}\right\}$ with respect to $C$. Since

$$
d\left(T x, x_{n}\right) \leq d\left(T x, T x_{n}\right)+d\left(T x_{n}, x_{n}\right) \leq d\left(x, x_{n}\right)+d\left(T x_{n}, x_{n}\right)
$$

we get

$$
\begin{aligned}
r\left(T x,\left\{x_{n}\right\}\right) & =\limsup _{n \rightarrow \infty} d\left(T x, x_{n}\right) \\
& \leq \limsup _{n \rightarrow \infty}\left[d\left(x, x_{n}\right)+d\left(T x_{n}, x_{n}\right)\right]=r\left(x,\left\{x_{n}\right\}\right) .
\end{aligned}
$$

By the uniqueness of the asymptotic center, we get $T x=x$.

The following theorem is necessary to discuss the behavior of the iterates in (2.1).

Theorem 4.1 Let $C$ be a nonempty, closed and convex subset of a complete uniformly convex hyperbolic space $X$. Let $S, T: C \rightarrow C$ be nonexpansive mappings such that $F \neq \emptyset$. Fix $x_{1} \in C$ and generate $\left\{x_{n}\right\}$ by (2.1). Set

$$
y_{n}=\beta_{n} T x_{n} \oplus\left(1-\beta_{n}\right) x_{n}
$$

for any $n \geq 1$.

(i) If $\alpha_{n} \in[a, b]$, where $0<a \leq b<1$, then

$$
\lim _{n \rightarrow \infty} d\left(x_{n}, S y_{n}\right)=0
$$

(ii) If $\liminf _{n \rightarrow \infty} \alpha_{n}>0$ and $\beta_{n} \in[a, b]$, with $0<a \leq b<1$, then

$$
\lim _{n \rightarrow \infty} d\left(x_{n}, T x_{n}\right)=0
$$

(iii) If $\alpha_{n}, \beta_{n} \in[a, b]$, with $0<a \leq b<1$, then

$$
\lim _{n \rightarrow \infty} d\left(x_{n}, S x_{n}\right)=0 \quad \text { and } \quad \lim _{n \rightarrow \infty} d\left(x_{n}, T x_{n}\right)=0
$$

Proof Let $p \in F$. Then the sequence $\left\{d\left(x_{n}, p\right)\right\}$ is decreasing. Set $c=\lim _{n \rightarrow \infty} d\left(x_{n}, p\right)$. If $c=0$, then all the conclusions are trivial. Therefore we will assume that $c>0$. Note that 
we have

$$
d\left(x_{n+1}, p\right) \leq \alpha_{n} d\left(S y_{n}, p\right)+\left(1-\alpha_{n}\right) d\left(x_{n}, p\right)
$$

and

$$
d\left(S y_{n}, p\right) \leq d\left(y_{n}, p\right) \leq \beta_{n} d\left(T x_{n}, p\right)+\left(1-\beta_{n}\right) d\left(x_{n}, p\right) \leq d\left(x_{n}, p\right)
$$

for any $n \geq 1$. In order to prove (i), assume that $\alpha_{n} \in[a, b]$, where $0<a \leq b<1$. From the inequalities (4.1) and (4.2), we get

$$
d\left(x_{n+1}, p\right)=d\left(\alpha_{n} S y_{n} \oplus\left(1-\alpha_{n}\right) x_{n}, p\right) \leq \alpha_{n} d\left(S y_{n}, p\right)+\left(1-\alpha_{n}\right) d\left(x_{n}, p\right) \leq d\left(x_{n}, p\right),
$$

which implies $\lim _{n \rightarrow \infty} d\left(S y_{n}, p\right)=c$. Indeed, let $\mathcal{U}$ be an ultrafilter over $\mathbb{N}$. Then we have $\lim _{\mathcal{U}} \alpha_{n}=\alpha \in[a, b]$ and $\lim _{\mathcal{U}} d\left(x_{n}, p\right)=\lim _{\mathcal{U}} d\left(x_{n+1}, p\right)=c$. Hence

$$
c \leq \alpha \lim _{\mathcal{U}} d\left(S y_{n}, p\right)+(1-\alpha) c \leq c .
$$

Since $\alpha \neq 0$, we get $\lim _{\mathcal{U}} d\left(S y_{n}, p\right)=c$. Since $\mathcal{U}$ was an arbitrary ultrafilter, we get $\lim _{n \rightarrow \infty} d\left(S y_{n}, p\right)=c$ as claimed. Therefore we have

$$
\lim _{n \rightarrow \infty} d\left(x_{n}, p\right)=\lim _{n \rightarrow \infty} d\left(S y_{n}, p\right)=\lim _{n \rightarrow \infty} d\left(\alpha_{n} S y_{n} \oplus\left(1-\alpha_{n}\right) x_{n}, p\right)=c .
$$

Using Lemma 2.5, we get $\lim _{n \rightarrow \infty} d\left(S y_{n}, x_{n}\right)=0$.

Next we prove (ii). Assume $\liminf _{n \rightarrow \infty} \alpha_{n}>0$ and $\beta_{n} \in[a, b]$, with $0<a \leq b<1$. First note that from (4.1) and (4.2), we get

$$
d\left(x_{n+1}, p\right) \leq \alpha_{n} d\left(y_{n}, p\right)+\left(1-\alpha_{n}\right) d\left(x_{n}, p\right) \leq d\left(x_{n}, p\right),
$$

which implies $\lim _{n \rightarrow \infty} \alpha_{n} d\left(y_{n}, p\right)+\left(1-\alpha_{n}\right) d\left(x_{n}, p\right)=c$. Since $\liminf _{n \rightarrow \infty} \alpha_{n}>0$, we conclude that $\lim _{n \rightarrow \infty} d\left(y_{n}, p\right)=c$. Since $\beta_{n} \geq a>0$, we get in a similar fashion $\lim _{n \rightarrow \infty} d\left(T x_{n}, p\right)=c$. Therefore we have

$$
\lim _{n \rightarrow \infty} d\left(x_{n}, p\right)=\lim _{n \rightarrow \infty} d\left(T x_{n}, p\right)=\lim _{n \rightarrow \infty} d\left(\beta_{n} T x_{n} \oplus\left(1-\beta_{n}\right) x_{n}, p\right)=c
$$

Using Lemma 2.5, we get $\lim _{n \rightarrow \infty} d\left(T x_{n}, x_{n}\right)=0$.

Finally let us prove (iii). Assume that $\alpha_{n}, \beta_{n} \in[a, b]$, with $0<a \leq b<1$. Then from (i) and (ii), we know that

$$
\lim _{n \rightarrow \infty} d\left(x_{n}, S y_{n}\right)=0 \quad \text { and } \quad \lim _{n \rightarrow \infty} d\left(x_{n}, T x_{n}\right)=0
$$

Since

$$
\begin{aligned}
d\left(x_{n}, S x_{n}\right) & \leq d\left(x_{n}, S y_{n}\right)+d\left(S y_{n}, S x_{n}\right) \\
& \leq d\left(x_{n}, S y_{n}\right)+d\left(y_{n}, x_{n}\right)
\end{aligned}
$$




$$
\begin{aligned}
& =d\left(x_{n}, S y_{n}\right)+\beta_{n} d\left(T x_{n}, x_{n}\right) \\
& \leq d\left(x_{n}, S y_{n}\right)+d\left(T x_{n}, x_{n}\right),
\end{aligned}
$$

we conclude that $\lim _{n \rightarrow \infty} d\left(x_{n}, S x_{n}\right)=0$.

The conclusion of Theorem 4.1(iii) is amazing because the sequence generated by (2.1) gives an approximate fixed point sequence for both $S$ and $T$ without assuming that these mappings commute.

Remark 4.1 If we assume that $0 \leq \beta_{n} \leq b<1$ and $\liminf _{n \rightarrow \infty} \alpha_{n}>0$, then

$$
\lim _{n \rightarrow \infty} \beta_{n} d\left(x_{n}, T x_{n}\right)=0 .
$$

Indeed, if we assume this not to be so, then there exists a subsequence $\left\{\beta_{n^{\prime}}\right\}$ and $\delta>0$ such that

$$
\beta_{n^{\prime}} d\left(x_{n^{\prime}}, T x_{n^{\prime}}\right) \geq \delta
$$

for any $n \geq 1$. In particular, it is clear, since $\left\{d\left(x_{n}, T x_{n}\right)\right\}$ is bounded, that $\lim _{n \rightarrow \infty} \beta_{n^{\prime}} \neq 0$. Without loss of generality, we may assume that $\beta_{n^{\prime}} \geq a>0$, for $n \geq 1$. The proof of (ii) will imply

$$
\lim _{n \rightarrow \infty} d\left(x_{n^{\prime}}, T x_{n^{\prime}}\right)=0
$$

which is a contradiction since $\left\{\beta_{n}\right\}$ is a bounded sequence. Therefore we must have

$$
\lim _{n \rightarrow \infty} \beta_{n} d\left(x_{n}, T x_{n}\right)=0
$$

In particular, if we assume $\alpha_{n} \in[a, b]$, then we get

$$
\lim _{n \rightarrow \infty} d\left(x_{n}, S x_{n}\right)=0
$$

As a direct consequence to Theorem 4.1 and Remark 4.1, we get the following result which discusses the $\Delta$-convergence of the iterative sequence defined by (2.1).

Theorem 4.2 Let $C$ be a nonempty, closed and convex subset of a complete uniformly convex hyperbolic space $X$. Let $S, T: C \rightarrow C$ be two nonexpansive mappings such that $F \neq \emptyset$. Fix $x_{1} \in C$ and generate $\left\{x_{n}\right\}$ by (2.1). Then

(i) if $\alpha_{n} \in[a, b]$ and $\beta_{n} \in[0, b]$, with $0<a \leq b<1$, then $x_{n} \stackrel{\Delta}{\rightarrow} y$ and $y \in F(S)$;

(ii) if $\alpha_{n} \in[a, 1]$ and $\beta_{n} \in[a, b]$, with $0<a \leq b<1$, then $x_{n} \stackrel{\Delta}{\rightarrow} y$ and $y \in F(T)$;

(iii) if $\alpha_{n}, \beta_{n} \in[a, b]$, with $0<a \leq b<1$, then $x_{n} \stackrel{\Delta}{\rightarrow} y$ and $y \in F$.

Proof Let us prove (i). Assume $\alpha_{n} \in[a, b]$ and $\beta_{n} \in[0, b]$, with $0<a \leq b<1$. Theorem 4.1 and Remark 4.1 imply that $\left\{x_{n}\right\}$ is an approximate fixed point sequence of $S$, i.e.,

$$
\lim _{n \rightarrow \infty} d\left(x_{n}, S x_{n}\right)=0
$$


Let $y$ be the unique asymptotic center of $\left\{x_{n}\right\}$. Then Lemma 4.1 implies that $y \in F(S)$. Let us prove that in fact $\left\{x_{n}\right\} \Delta$-converges to $y$. Let $\left\{x_{n_{i}}\right\}$ be a subsequence of $\left\{x_{n}\right\}$. Let $z$ be the unique asymptotic center of $\left\{x_{n_{i}}\right\}$. Again since $\left\{x_{n_{i}}\right\}$ is an approximate fixed point sequence of $S$, we get $z \in F(S)$. Hence

$$
\limsup _{n_{i} \rightarrow \infty} d\left(x_{n_{i}}, z\right) \leq \limsup _{n_{i} \rightarrow \infty} d\left(x_{n_{i}}, y\right) .
$$

Since $y, z \in F(S)$, we get

$$
\limsup _{n_{i} \rightarrow \infty} d\left(x_{n_{i}}, z\right)=\lim _{n \rightarrow \infty} d\left(x_{n}, z\right) \quad \text { and } \quad \limsup _{n_{i} \rightarrow \infty} d\left(x_{n_{i}}, y\right)=\lim _{n \rightarrow \infty} d\left(x_{n}, y\right) .
$$

Since $y$ is the unique asymptotic center of $\left\{x_{n}\right\}$, we get $y=z$. This proves that $\left\{x_{n}\right\} \Delta$-converges to $y$.

Next we prove (ii). Assume $\alpha_{n} \in[a, 1]$ and $\beta_{n} \in[a, b]$, with $0<a \leq b<1$. Then Theorem 4.1 implies that $\left\{x_{n}\right\}$ is an approximate fixed point sequence of $T$, i.e.,

$$
\lim _{n \rightarrow \infty} d\left(x_{n}, T x_{n}\right)=0
$$

Following the same proof as given above for (i), we get $\left\{x_{n}\right\} \Delta$-converges to its unique asymptotic center which is a fixed point of $T$.

The conclusion (iii) follows easily from (i) and (ii).

As a corollary to Theorem 4.2, we get the following result when $S=T$.

Corollary 4.1 Let $C$ be a nonempty, closed and convex subset of a complete uniformly convex hyperbolic space $X$. Let $T: C \rightarrow C$ be a nonexpansive mapping with a fixed point. Suppose that $\left\{x_{n}\right\}$ is given by (2.2), where $\alpha_{n} \in[a, b]$ and $\beta_{n} \in[0, b]$ or $\alpha_{n} \in[a, 1]$ and $\beta_{n} \in$ $[a, b]$, with $0<a \leq b<1$. Then $x_{n} \stackrel{\Delta}{\rightarrow} p$, with $p \in F(T)$.

Using the concept of near point projection, we establish the following amazing convergence result.

Theorem 4.3 Let $C$ be a nonempty, closed and convex subset of a complete uniformly convex hyperbolic space $X$. Let $S, T: C \rightarrow C$ be nonexpansive mappings such that $F \neq \emptyset$. Let $P$ be the nearest point projection of $C$ onto $F$. For an initial value $x_{1} \in C$, define $\left\{x_{n}\right\}$ as given in (2.1), where $\alpha_{n}, \beta_{n} \in[a, b]$, with $0<a \leq b<1$. Then $\left\{P x_{n}\right\}$ converges strongly to the asymptotic center of $\left\{x_{n}\right\}$.

Proof First, we claim that

$$
d\left(P x_{n}, x_{n+m}\right) \leq d\left(P x_{n}, x_{n}\right) \quad \text { for } m \geq 1, n \geq 1 .
$$

We prove (4.3) by induction on $m \geq 1$. For $m=1$, we have

$$
\begin{aligned}
d\left(P x_{n}, x_{n+1}\right) & =d\left(P x_{n}, \alpha_{n} S y_{n} \oplus\left(1-\alpha_{n}\right) x_{n}\right) \\
& \leq \alpha_{n} d\left(P x_{n}, S y_{n}\right)+\left(1-\alpha_{n}\right) d\left(P x_{n}, x_{n}\right)
\end{aligned}
$$




$$
\begin{aligned}
& \leq \alpha_{n} d\left(P x_{n}, y_{n}\right)+\left(1-\alpha_{n}\right) d\left(P x_{n}, x_{n}\right) \\
& =\alpha_{n} d\left(P x_{n}, \beta_{n} T x_{n} \oplus\left(1-\beta_{n}\right) x_{n}\right)+\left(1-\alpha_{n}\right) d\left(P x_{n}, x_{n}\right) \\
& \leq \alpha_{n}\left[\beta_{n} d\left(P x_{n}, T x_{n}\right)+\left(1-\beta_{n}\right) d\left(P x_{n}, x_{n}\right)\right]+\left(1-\alpha_{n}\right) d\left(P x_{n}, x_{n}\right) \\
& \leq \alpha_{n}\left[\beta_{n} d\left(P x_{n}, x_{n}\right)+\left(1-\beta_{n}\right) d\left(P x_{n}, x_{n}\right)\right]+\left(1-\alpha_{n}\right) d\left(P x_{n}, x_{n}\right) \\
& =d\left(P x_{n}, x_{n}\right)
\end{aligned}
$$

That is,

$$
d\left(P x_{n}, x_{n+1}\right) \leq d\left(P x_{n}, x_{n}\right)
$$

for $n \geq 1$. Assume that (4.3) is true for $m=k$. That is,

$$
d\left(P x_{n}, x_{n+k}\right) \leq d\left(P x_{n}, x_{n}\right)
$$

for $n \geq 1$. Hence

$$
\begin{aligned}
d\left(P x_{n}, x_{n+k+1}\right)= & d\left(P x_{n}, \alpha_{n+k} S y_{n+k} \oplus\left(1-\alpha_{n+k}\right) x_{n+k}\right) \\
\leq & \alpha_{n+k} d\left(P x_{n}, S y_{n+k}\right)+\left(1-\alpha_{n+k}\right) d\left(P x_{n}, x_{n+k}\right) \\
\leq & \alpha_{n+k} d\left(P x_{n}, y_{n+k}\right)+\left(1-\alpha_{n+k}\right) d\left(P x_{n}, x_{n+k}\right) \\
= & \alpha_{n+k} d\left(P x_{n}, \beta_{n+k} T x_{n+k} \oplus\left(1-\beta_{n+k}\right) x_{n+k}\right) \\
& +\left(1-\alpha_{n+k}\right) d\left(P x_{n}, x_{n+k}\right) \\
\leq & \alpha_{n+k}\left[\beta_{n+k} d\left(P x_{n}, T x_{n+k}\right)+\left(1-\beta_{n+k}\right) d\left(P x_{n}, x_{n+k}\right)\right] \\
& +\left(1-\alpha_{n+k}\right) d\left(P x_{n}, x_{n+k}\right) \\
\leq & \alpha_{n+k}\left[\beta_{n+k} d\left(P x_{n}, x_{n+k}\right)+\left(1-\beta_{n+k}\right) d\left(P x_{n}, x_{n+k}\right)\right] \\
& +\left(1-\alpha_{n+k}\right) d\left(P x_{n}, x_{n+k}\right) \\
= & d\left(P x_{n}, x_{n+k}\right) \\
\leq & d\left(P x_{n}, x_{n}\right) .
\end{aligned}
$$

This completes the proof of (4.3). Let us complete the proof of Theorem 4.3. We know from Theorem 4.2(iii) that $\left\{x_{n}\right\} \Delta$-converges to its unique asymptotic center $y$, which is in $F$. Let us prove that $\left\{P x_{n}\right\}$ converges strongly to $y$. Assume not, i.e., there exist $\varepsilon>0$ and a subsequence $\left\{P x_{n_{i}}\right\}$ such that $d\left(P x_{n_{i}}, y\right) \geq \varepsilon$, for any $n_{i} \geq 1$. It is clear that we must have $R=d\left(x_{1}, y\right)>0$, otherwise $\left\{x_{n}\right\}$ is a constant sequence. Since

$$
\left\{\begin{array}{l}
d\left(x_{n_{i}}, y\right) \leq d\left(x_{n_{i}}, y\right) \\
d\left(x_{n_{i}}, P x_{n_{i}}\right) \leq d\left(x_{n_{i}}, y\right) \\
d\left(P x_{n_{i}}, y\right) \geq \varepsilon=d\left(x_{n_{i}}, y\right) \frac{\varepsilon}{d\left(x_{n_{i}}, y\right)} \geq d\left(x_{n_{i}}, y\right) \frac{\varepsilon}{R}
\end{array}\right.
$$

we get

$$
d\left(x_{n_{i}}, \frac{1}{2} P x_{n_{i}} \oplus \frac{1}{2} y\right) \leq d\left(x_{n_{i}}, y\right)\left(1-\delta\left(d\left(x_{n_{i}}, y\right), \frac{\varepsilon}{R}\right)\right)
$$


for any $n_{i} \geq 1$. Using the properties of the modulus of uniform convexity, there exists $\eta>0$ such that

$$
\delta\left(d\left(x_{n_{i}}, y\right), \frac{\varepsilon}{R}\right) \geq \eta
$$

for any $n_{i} \geq 1$. Hence

$$
d\left(x_{n_{i}}, \frac{1}{2} P x_{n_{i}} \oplus \frac{1}{2} y\right) \leq d\left(x_{n_{i}}, y\right)(1-\eta)
$$

for any $n_{i} \geq 1$. Using the definition of the nearest point projection $P$, we get

$$
d\left(x_{n_{i}}, P x_{n_{i}}\right) \leq d\left(x_{n_{i}}, y\right)(1-\eta)
$$

for any $n_{i} \geq 1$. Using the inequality (4.3) above, we get

$$
d\left(x_{n_{i}+m}, P x_{n_{i}}\right) \leq d\left(x_{n_{i}}, y\right)(1-\eta)
$$

for any $n_{i} \geq 1$ and $m \geq 1$. Since $P x_{n_{i}} \in F$, we know that $\left\{d\left(x_{n}, P x_{n_{i}}\right)\right\}$ is decreasing (in $n$ and fixed $\left.n_{i}\right)$. Hence

$$
\limsup _{m \rightarrow \infty} d\left(x_{n_{i}+m}, P x_{n_{i}}\right)=\lim _{n \rightarrow \infty} d\left(x_{n}, P x_{n_{i}}\right) \leq d\left(x_{n_{i}}, y\right)(1-\eta)
$$

for any $n_{i} \geq 1$. Since $y$ is the asymptotic center of $\left\{x_{n}\right\}$, we get

$$
\lim _{n \rightarrow \infty} d\left(x_{n}, y\right) \leq \lim _{n \rightarrow \infty} d\left(x_{n}, P x_{n_{i}}\right) \leq d\left(x_{n_{i}}, y\right)(1-\eta)
$$

for any $n_{i} \geq 1$. Finally since $y \in F$, if we let $n_{i} \rightarrow \infty$, we get

$$
\lim _{n \rightarrow \infty} d\left(x_{n}, y\right) \leq \lim _{n \rightarrow \infty} d\left(x_{n}, y\right)(1-\eta)
$$

Since $\varepsilon \leq d\left(x_{n_{i}}, P x_{n_{i}}\right) \leq d\left(x_{n_{i}}, y\right)$, we conclude that $\varepsilon \leq \lim _{n \rightarrow \infty} d\left(x_{n}, y\right)$, which implies $1 \leq$ $1-\eta$ which is our desired contradiction. Therefore $\left\{P x_{n}\right\}$ converges strongly to $y$.

\section{Competing interests}

The authors declare that they have no competing interests.

Authors' contributions

All authors contributed equally to the writing of this paper. All authors read and approved the final manuscript.

\section{Author details}

${ }^{1}$ Department of Mathematics and Statistics, King Fahd University of Petroleum and Minerals, Dhahran, 31261, Saudi Arabia. ${ }^{2}$ Department of Mathematics, The Islamia University of Bahawalpur, Bahawalpur, 63100, Pakistan. ${ }^{3}$ Department of Mathematical Sciences, University of Texas at El Paso, El Paso, TX 79968, USA

\section{Acknowledgements}

The authors are grateful to King Fahd University of Petroleum and Minerals for supporting research project IN121055. 


\section{References}

1. Das, G, Debata, P: Fixed points of quasi-nonexpansive mappings. Indian J. Pure Appl. Math. 17, 1263-1269 (1986)

2. Takahashi, W, Tamura, T: Convergence theorems for a pair of nonexpansive mappings. J. Convex Anal. 5, 45-56 (1998)

3. Khan, SH, Fukhar-ud-din, $\mathrm{H}$ : Weak and strong convergence of a scheme with errors for two nonexpansive mappings. Nonlinear Anal. 61, 1295-1301 (2005)

4. Takahashi, W, Kim, GE: Approximating fixed points of nonexpansive mappings in Banach spaces. Math. Jpn. 48, 1-9 (1998)

5. Kirk, WA: An abstract fixed point theorem for nonexpansive mappings. Proc. Am. Math. Soc. 82, 640-642 (1981)

6. Kirk, WA: Fixed point theory for nonexpansive mappings II. Contemp. Math. 18, 121-140 (1983)

7. Penot, JP: Fixed point theorems without convexity. Bull. Soc. Math. Fr. 60, 129-152 (1979)

8. Takahashi, W: A convexity in metric spaces and nonexpansive mappings. Kodai Math. Semin. Rep. 22, 142-149 (1970)

9. Menger, K: Untersuchungen über allgemeine Metrik. Math. Ann. 100, 75-163 (1928)

10. Borwein, J, Reich, S, Shafrir, I: Krasnoselski-Mann iterations in normed spaces. Can. Math. Bull. 35, 21-28 (1992)

11. Reich, S, Shafrir, I: Nonexpansive iterations in hyperbolic spaces. Nonlinear Anal. 15, 537-558 (1990)

12. Khan, AR, Fukhar-ud-din, H, Khan, MAA: An implicit algorithm for two finite families of nonexpansive maps in hyperbolic spaces. Fixed Point Theory Appl. 2012, 54 (2012)

13. Kohlenbach, U: Some logical metatheorems with applications in functional analysis. Trans. Am. Math. Soc. 357, 89-128 (2005)

14. Busemann, H: Spaces with non-positive curvature. Acta Math. 80, 259-310 (1948)

15. Goebel, K, Reich, S: Uniform Convexity, Hyperbolic Geometry, and Nonexpansive Mappings. Series of Monographs and Textbooks in Pure and Applied Mathematics, vol. 83. Dekker, New York (1984)

16. Kirk, WA: A fixed point theorem in CAT(0) spaces and $\mathbb{R}$-trees. Fixed Point Theory Appl. 2004(4), $309-316$ (2004)

17. Leustean, L: A quadratic rate of asymptotic regularity for CAT(0)-spaces. J. Math. Anal. Appl. 325, 386-399 (2007)

18. Bridson, M, Haefliger, A: Metric Spaces of Non-Positive Curvature. Springer, Berlin (1999)

19. Dhompongsa, S, Panyanak, B: On $\Delta$-convergence theorems in CAT(0) spaces. Comput. Math. Appl. 56, 2572-2579 (2008)

20. Khan, AR, Khamsi, MA, Fukhar-ud-din, H: Strong convergence of a general iteration scheme in CAT(0)-spaces. Nonlinear Anal. 74, 783-791 (2011)

21. Shimizu, T, Takahashi, W: Fixed points of multivalued mappings in certain convex metric spaces. Topol. Methods Nonlinear Anal. 8, 197-203 (1996)

22. Ibn Dehaish, BA, Khamsi, MA, Khan, AR: Mann iteration process for asymptotic pointwise nonexpansive mappings in metric spaces. J. Math. Anal. Appl. 397, 861-868 (2013)

23. Fukhar-ud-din, $H$, Khan, AR, Akhtar, Z: Fixed point results for a generalized nonexpansive map in uniformly convex metric spaces. Nonlinear Anal. 75, 4747-4760 (2012)

24. Bruhat, F, Tits, J: Groupes réductifs sur un corps local. I. Données radicielles valuées. Publ. Math. IHES 41, 5-251 (1972)

25. Khamsi, MA, Khan, AR: Inequalities in metric spaces with applications. Nonlinear Anal. 74, 4036-4045 (2011)

26. Kuczumow, T: An almost convergence and its applications. Ann. Univ. Mariae Curie-Skłodowska, Sect. A 32, 79-88 (1978)

27. Lim, TC: Remarks on some fixed point theorems. Proc. Am. Math. Soc. 60, 179-182 (1976)

28. Fukhar-ud-din, $\mathrm{H}$ : Existence and approximation of fixed points in convex metric spaces. Carpath. J. Math. (to appear)

29. Shimizu, T: A convergence theorem to common fixed points of families of nonexpansive mappings in convex metric spaces. In: Proceedings of the International Conference on Nonlinear and Convex Analysis, pp. 575-585 (2005)

30. Göhde, D: Zum Prinzip der kontraktiven Abbildung. Math. Nachr. 30, 251-258 (1965)

10.1186/1687-1812-2014-113

Cite this article as: Fukhar-ud-din and Khamsi: Approximating common fixed points in hyperbolic spaces. Fixed Point Theory and Applications 2014, 2014:113

\section{Submit your manuscript to a SpringerOpen ${ }^{\ominus}$ journal and benefit from:}

- Convenient online submission

- Rigorous peer review

- Immediate publication on acceptance

- Open access: articles freely available online

- High visibility within the field

- Retaining the copyright to your article 